\title{
Analytic model of phononic crystals with local resonances
}

\author{
Zhengyou Liu, ${ }^{1,2}$ C. T. Chan, ${ }^{1}$ and Ping Sheng ${ }^{1}$ \\ ${ }^{1}$ Department of Physics, The Hong Kong University of Science and Technology, Clear Water Bay, Hong Kong, China \\ ${ }^{2}$ Department of Physics, Wuhan University, Wuhan 430072, China
}

(Received 19 April 2003; revised manuscript received 27 September 2004; published 3 January 2005)

\begin{abstract}
A simple analytic model is presented to describe the low-frequency effective mass densities of threecomponent phononic crystals with local resonances. We show that the effective mass densities can turn negative close to the local resonances. Expressions for the effective mass densities are derived for both threedimensional systems with coated spheres embedded in a host matrix, and two-dimensional systems with coated cylinders embedded in a host matrix.
\end{abstract}

DOI: 10.1103/PhysRevB.71.014103

PACS number(s): 43.20.+g, 43.35. $+\mathrm{d}, 43.40 .+\mathrm{s}$

\section{INTRODUCTION}

A recent experiment ${ }^{1}$ showed that certain threecomponent composites, consisting of hard spheres coated with a soft cladding and dispersed in a stiff host medium, exhibit complete elastic wave band gaps. These spectral gaps arise from local vibration resonances of the coated spheres, and have nothing to do with Bragg scattering. ${ }^{1}$ If the cladding is very soft, the band-gap frequency can be very low, making such a composite an interesting material for blocking low-frequency sound. Such an anomalous response of the system is the consequence of the coupling of the longwavelength elastic wave in the host medium with the localized vibrational motion of the spheres. Such behavior is rather analogous to the coupling of electromagnetic waves with transverse optical phonons in an ionic crystal, leading to a polariton gap and an effective negative dielectric constants for near the resonance frequencies. ${ }^{2}$ Similar results were reported for a three-component two-dimensional (2D) system: resonance-induced band gap was observed for rubber-coated lead cylinders embedded in an epoxy matrix. ${ }^{3}$ For $2 \mathrm{D}$ structures formed with rubber-coated square lead rods embedded in an epoxy matrix, numerical simulations indicate that the transmission coefficient show dips at resonant frequencies. Dips in transmission loss spectra of stiff structures containing local resonant units can be modeled by simple onedimensional harmonic oscillators with frequency-dependent effective complex mass. ${ }^{4}$ While the three-dimensional (3D) system with rubber-coated lead spheres embedded in epoxy can be described with full mathematical rigor using a multiple scattering approach, ${ }^{5}$ we show here that the essence of the physics can be captured in a simple analytic model. Such an analytic model is highly desirable for an intuitive understanding of the problem, as well as offering a quick and yet reasonably good estimate of the effective properties of such a complex system. This simple physical model is designed to describe the three-component composite material with the special configuration: hard spheres (or cylinders) coated with soft material distributed uniformly (i.e., randomly or periodically) in a stiff matrix. It turns out that when the spheres and the matrix are much harder than the coating, analytic formulae that describe the effective mass density (EMD) of the material that display resonance can be derived. It has been shown previously ${ }^{1}$ that the low-frequency band gap was in- duced by negative response functions at the band-gap frequency. Since the sound speed is proportional to $\sqrt{\kappa / \rho}$, where $\kappa$ and $\rho$ are modulus and density, respectively, a negative $\kappa / \rho$ implies an exponential wave attenuation. Here, we describe the models for both $3 \mathrm{D}$ and 2D systems of such structures, by calculating their effective mass density, and show that EMD becomes negative near the resonance frequencies, giving rise to exponential attenuation of wave. When the resonance behavior is properly described, we find that these structures behave like dispersive media as expected, having frequency dependent EMDs. This is in contrast to the ordinary composite structures without local resonances. In that case, the EMDs of the structures are simply the arthimatic averages of the mass densities of the components. In the long wavelength limit away from the resonance frequencies, the EMDs reduce to the arthimatic averages. The negative EMD is seen to result from the coupling of traveling waves with the local resonances. In particular, the local resonances enable the strong coupling even though the size of the locally resonant unit is much smaller than the wavelength in the matrix, in contrast to the Raleigh scattering case. In what follows, we first consider the 3D systems in Sec. II, followed by the 2D case in Sec. III. A brief summary is presented in Sec. IV. The mathematical details for the 2D case are presented in the Appendix.

\section{THREE-DIMENSIONAL SYSTEMS}

Consider a 3D three-component composite model consisting of a collection of hard spheres, each coated with a soft material, embedded in a matrix material. The basic building block is shown schematically in Fig. 1, where the matrix medium is labeled 1 , the coating medium labeled 2 , and the hard-core sphere labeled 3 . The inner and outer radii of the coating layer are denoted with $a$ and $b$, respectively. Because of the softness of the coating medium, the hard-core sphere is hardly deformed, and thus can be treated approximately as being rigid. We are interested in wave propagation with a wavelength that is very large compared with the dimension of the inclusions. Subjected to a long-wavelength incident wave (either longitudinal or transverse), the movement of the spherical core (as well as that of the matrix 1 locally) can be regarded as a vibration around the center of the sphere. Sup- 


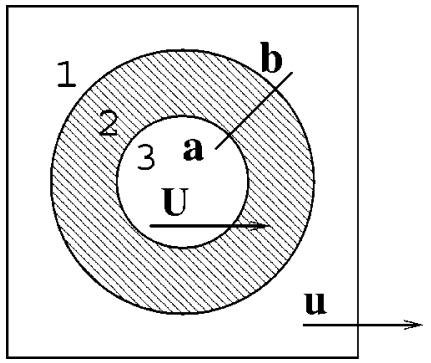

FIG. 1. A basic unit in the three component composite material. Mediums 1 and 3 are hard materials, and medium 2 is a soft material. The arrows indicate the vibration directions for mediums 1 and 3 , respectively.

pose that the vibration is along the $z$ direction (marked by the arrow in Fig. 1), the equation of motion for the sphere may be expressed as

$$
-m_{3} \omega^{2} U=\iint_{s}\left(\tau_{r r} \cos \theta-\tau_{r \theta} \sin \theta\right) a^{2} \sin \theta d \theta d \phi,
$$

where $U$ is the displacement of the sphere, $m_{3}$ is the mass of the sphere, $\tau_{r r}$ and $\tau_{r \theta}$ are stress components in medium 2 with $r, \theta$, and $\phi$, denoting the spherical coordinates. The integration in the equation is performed on the surface of the sphere. In medium 2, the motion of the medium satisfies the elastic wave equation, ${ }^{6}$ given by

$$
\left(\lambda_{2}+2 \mu_{2}\right) \boldsymbol{\nabla}(\boldsymbol{\nabla} \cdot \mathbf{u})-\mu_{2} \boldsymbol{\nabla} \times \boldsymbol{\nabla} \times \mathbf{u}+\rho_{2} \omega^{2} \mathbf{u}=0,
$$

where $\lambda_{2}$ and $\mu_{2}$ are the Lamé constants, and $\rho_{2}$ is the mass density of medium 2 . The azimuthal symmetry of the building block implies that displacements may be expressed as ${ }^{7}$

$$
\vec{u}=u_{r} \vec{e}_{r}+u_{\theta} \vec{e}_{\theta}
$$

or

$$
\vec{u}=\nabla \Phi+\nabla \times\left(\frac{\partial \Psi}{\partial \theta} \vec{e}_{\phi}\right),
$$

where $\Phi$ and $\Psi$ are scalar potential functions, satisfying the following scalar wave equations:

$$
\begin{aligned}
& \nabla^{2} \Phi+\alpha^{2} \Phi=0, \\
& \nabla^{2} \Psi+\beta^{2} \Psi=0,
\end{aligned}
$$

where $\alpha=\omega \sqrt{\rho_{2} /\left(\lambda_{2}+2 \mu_{2}\right)}$, and $\beta=\omega \sqrt{\rho_{2} / \mu_{2}}$. The solutions for $\Phi$ and $\Psi$ may be written as

$$
\begin{aligned}
& \Phi=\sum_{n=0}^{\infty}\left[A_{n} j_{n}(\alpha r)+B_{n} n_{n}(\alpha r)\right] P_{n}(\cos \theta), \\
& \Psi=\sum_{n=0}^{\infty}\left[C_{n} j_{n}(\beta r)+D_{n} n_{n}(\beta r)\right] P_{n}(\cos \theta),
\end{aligned}
$$

where $j_{n}(x)$ is the $n$th order spherical Bessel function, $n_{n}(x)$ is the $n$th order spherical Neumann function, and $P_{n}(x)$ is the $n$th order Legendre polynomial. With $u$ denoting the local displacement of medium 3 (see Fig. 1), by regarding the core sphere and the matrix as almost rigid, continuity across the interface requires that

$$
\begin{aligned}
& \left.u_{r}\right|_{r=b}=u \cos \theta, \\
& u_{\left.\theta\right|_{r=b}}=-u \sin \theta, \\
& u_{\left.r\right|_{r=a}}=U \cos \theta, \\
& \left.u_{\theta}\right|_{r=a}=-U \sin \theta,
\end{aligned}
$$

which means that the expansions in Eqs. (7) and (8) actually truncates for $n>1$. That leads to a set of linear equations

$$
\begin{aligned}
& E_{11} A_{1}+E_{12} B_{1}+E_{13} C_{1}+E_{14} D_{1}=b u, \\
& E_{21} A_{1}+E_{22} B_{1}+E_{23} C_{1}+E_{24} D_{1}=b u, \\
& E_{31} A_{1}+E_{32} B_{1}+E_{33} C_{1}+E_{34} D_{1}=a U, \\
& E_{41} A_{1}+E_{42} B_{1}+E_{43} C_{1}+E_{44} D_{1}=a U,
\end{aligned}
$$

where $E_{i j}$ s are given by

$$
\begin{gathered}
E_{11}=j_{1}(\alpha b)-\alpha b j_{2}(\alpha b), \\
E_{12}=n_{1}(\alpha b)-\alpha b n_{2}(\alpha b), \\
E_{13}=-2 j_{1}(\beta b), \\
E_{14}=-2 n_{1}(\beta b), \\
E_{21}=j_{1}(\alpha b), \\
E_{22}=n_{1}(\alpha b), \\
E_{23}=-2 j_{1}(\beta b)+\beta b j_{2}(\beta b), \\
E_{24}=-2 n_{1}(\beta b)+\beta b n_{2}(\beta b), \\
E_{31}=j_{1}(\alpha a)-\alpha a j_{2}(\alpha a), \\
E_{32}=n_{1}(\alpha a)-\alpha a n_{2}(\alpha a), \\
E_{43}=-2 j_{1}(\beta a)+\beta a j_{2}(\beta a), \\
E_{33}=-2 j_{1}(\beta a), \\
E_{34}=-2 n_{1}(\beta a), \\
E_{41}=j_{1}(\alpha a), \\
E_{42}=n_{1}(\alpha a), \beta a n_{2}(\beta a) .
\end{gathered}
$$

Let us denote the inverse of matrix $E$ by $T$, then we get

$$
A_{1}=\left(T_{11}+T_{12}\right) b u+\left(T_{13}+T_{14}\right) a U,
$$




$$
\begin{aligned}
& B_{1}=\left(T_{21}+T_{22}\right) b u+\left(T_{23}+T_{24}\right) a U, \\
& C_{1}=\left(T_{31}+T_{32}\right) b u+\left(T_{33}+T_{34}\right) a U, \\
& D_{1}=\left(T_{41}+T_{42}\right) b u+\left(T_{43}+T_{44}\right) a U .
\end{aligned}
$$

Since the strain tensor may be expressed in terms of the displacement vector as ${ }^{6}$

$$
\boldsymbol{\epsilon}=\frac{1}{2}(\boldsymbol{\nabla} \vec{u}+\vec{u} \boldsymbol{\nabla}),
$$

and the stress tensor and the strain tensor are related by the law of elasticity

$$
\tau_{\zeta \xi}=\lambda_{2}\left(\epsilon_{r r}+\epsilon_{\theta \theta}+\epsilon_{z z}\right) \delta_{\zeta \xi}+2 \mu_{2} \epsilon_{\zeta \xi},
$$

we can obtain $\tau_{r r}$ and $\tau_{r \theta}$, and then the right-hand side of Eq. (1). Finally, we obtained the displacement of the sphere as

$$
U=-\frac{\frac{b}{a} g(\omega)}{R(\omega)-\frac{\rho_{3}}{\rho_{2}}} u,
$$

where $\rho_{3}$ is the density of the sphere, and the frequency dependent $g(\omega)$ and $R(\omega)$ are given, respectively, as

$$
\begin{aligned}
g(\omega)= & \left(T_{11}+T_{12}\right) j_{1}(\alpha a)+\left(T_{21}+T_{22}\right) n_{1}(\alpha a) \\
& -2\left(T_{31}+T_{32}\right) j_{1}(\beta a)-2\left(T_{41}+T_{42}\right) n_{1}(\beta a), \\
R(\omega)= & \left(T_{13}+T_{14}\right) j_{1}(\alpha a)+\left(T_{23}+T_{24}\right) n_{1}(\alpha a) \\
& -2\left(T_{33}+T_{34}\right) j_{1}(\beta a)-2\left(T_{43}+T_{44}\right) n_{1}(\beta a) .
\end{aligned}
$$

Equation (23) relates the displacement of the sphere to the displacement of the embedding matrix and we see that when $R(\omega)=\rho_{3} / \rho_{2}$, resonance occurs.

The expressions for $A_{1}, B_{1}, C_{1}$, and $D_{1}$, which will be needed in the following, may be derived by substituting Eq. (23) into Eqs. (17)-(20)

$$
\begin{aligned}
& A_{1}=\left[T_{11}+T_{12}-\left(T_{13}+T_{14}\right) \frac{g(\omega)}{R(\omega)-\frac{\rho_{3}}{\rho_{2}}}\right] b u, \\
& B_{1}=\left[T_{21}+T_{22}-\left(T_{23}+T_{24}\right) \frac{g(\omega)}{R(\omega)-\frac{\rho_{3}}{\rho_{2}}}\right] b u \text {, } \\
& C_{1}=\left[T_{31}+T_{32}-\left(T_{33}+T_{34}\right) \frac{g(\omega)}{R(\omega)-\frac{\rho_{3}}{\rho_{2}}}\right] b u, \\
& D_{1}=\left[T_{41}+T_{42}-\left(T_{43}+T_{44}\right) \frac{g(\omega)}{R(\omega)-\frac{\rho_{3}}{\rho_{2}}}\right] b u .
\end{aligned}
$$

The force acting on the coated sphere by the embedding matrix (medium 1) is given by

$$
F_{23}=\iint_{s}\left(\tau_{r r} \cos \theta-\tau_{r \theta} \sin \theta\right) b^{2} \sin \theta d \theta d \phi .
$$

After some manipulations, we get

$$
\begin{aligned}
F_{23}= & -\frac{4}{3} \pi b^{2} \rho_{2} \omega^{2}\left[A_{1} j_{1}(\alpha b)+B_{1} n_{1}(\alpha b)\right. \\
& \left.-2 C_{1} j_{1}(\beta b)-2 D_{1} n_{1}(\beta b)\right] \\
= & -\frac{4}{3} \pi b^{3} \rho_{2} \omega^{2}\left[g_{1}(\omega)-g_{2}(\omega) \frac{g(\omega)}{R(\omega)-\frac{\rho_{3}}{\rho_{2}}}\right] u,
\end{aligned}
$$

where

$$
\begin{aligned}
g_{1}(\omega)= & \left(T_{11}+T_{12}\right) j_{1}(\alpha b)+\left(T_{21}+T_{22}\right) n_{1}(\alpha b) \\
& -2\left(T_{31}+T_{32}\right) j_{1}(\beta b)-2\left(T_{41}+T_{42}\right) n_{1}(\beta b), \\
g_{2}(\omega)= & \left(T_{13}+T_{14}\right) j_{1}(\alpha b)+\left(T_{23}+T_{24}\right) n_{1}(\alpha b) \\
& -2\left(T_{33}+T_{34}\right) j_{1}(\beta b)-2\left(T_{43}+T_{44}\right) n_{1}(\beta b) .
\end{aligned}
$$

We note that the force between the coated sphere and the embedding medium, as expressed by $F_{23}$, is a consequence of the resonance behavior. Far away from the resonance and in the low frequency limit, $F_{23}$ reduces to $F_{23}^{0}=-\left(m_{2}\right.$ $\left.+m_{3}\right) \omega^{2} u$, with $m_{2}$ being the mass of the coating. Obviously $F_{23}^{0}$ is the force acting on the coated sphere by medium 1 if we ignore the resonance. We can define the effective mass density for the coated sphere through $F_{23}$ by

$$
-\rho_{23}^{e} V_{23} \omega^{2} u=F_{23},
$$

where $V_{23}=\frac{4}{3} \pi b^{3}$ is the volume of the coated sphere. We thus obtain

$$
\rho_{23}^{e}=\rho_{2}\left[g_{1}(\omega)-g_{2}(\omega) \frac{g(\omega)}{R(\omega)-\frac{\rho_{3}}{\rho_{2}}}\right] .
$$

The effective mass density for the whole local unit can be defined as

$$
\begin{aligned}
\rho_{e} & =\phi_{1} \rho_{1}+\left(\phi_{2}+\phi_{3}\right) \rho_{23}^{e} \\
& =\phi_{1} \rho_{1}+\left(\phi_{2}+\phi_{3}\right) \rho_{2}\left[g_{1}(\omega)-g_{2}(\omega) \frac{g(\omega)}{R(\omega)-\frac{\rho_{3}}{\rho_{2}}}\right]
\end{aligned}
$$

where $\phi_{1}, \phi_{2}$, and $\phi_{3}$ are the filling fractions for media 1,2 , and 3 , respectively, satisfying $\phi_{1}+\phi_{2}+\phi_{3}=1$. Since at the low-frequency limit $F_{23}$ reduces to $F_{23}^{0}$, we find that the effective mass density $\rho_{e}$ reduces to $\rho_{e}^{0}=\phi_{1} \rho_{1}+\phi_{2} \rho_{2}+\phi_{3} \rho_{3}$, which is the average density, i.e., the effective mass density if the local resonance is ignored. It should be noted that the force expressed in Eq. (31), which is obviously direction independent, is applicable only when the composite properties are isotropic at long wavelength. That requires the build- 

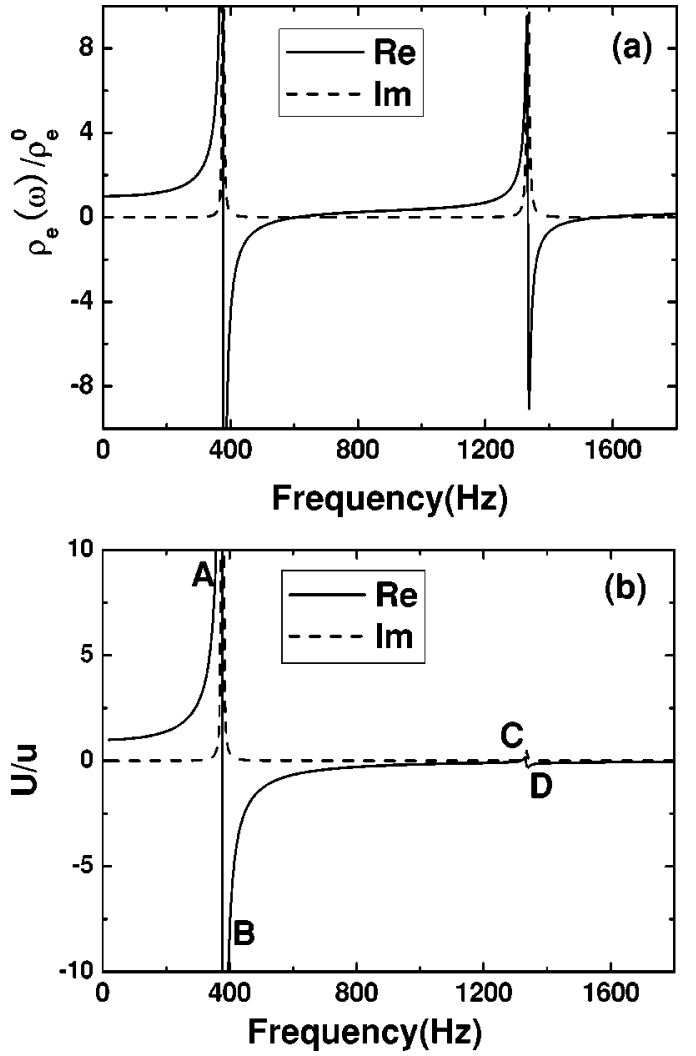

FIG. 2. (a) The $\operatorname{EMD(s)}$ and (b) the displacement of the core sphere for rubber-coated lead spheres embedded in an epoxy matrix. The solid line and dashed line represent the real part and imaginary part, respectively. The filling fraction for coated spheres is $47 \%$, the radius of the sphere is $5.0 \mathrm{~mm}$, and the coating thickness is $2.5 \mathrm{~mm}$. The displacement fields for the four frequencies labeled A, B, C, and D will be shown in Fig. 3 .

ing blocks to be randomly distributed, or arrayed with high symmetries, e.g., with cubic symmetries, so as to guarantee the effective medium's isotropy at long wavelength. Meanwhile, since the wavelengths in the matrix at the resonant frequencies are much greater than the size of the resonant unit, the longitudinal motion (when subjected to a longitudinal incident wave) and the transverse motion (when subjected to a transverse incident wave) of the unit are identical locally, so the same dispersion relation for the effective mass density is exhibited for the longitudial wave and the transverse wave.

To show that the effective mass density may become negative at some frequency regimes, we calculate the EMD for the three-component system discussed in Ref. 1: rubbercoated lead spheres embedded in epoxy matrix in a simple cubic structure. The filling fraction is $47 \%$, and the lead sphere radius $a$ is $0.5 \mathrm{~cm}$ and the thickness of the rubber coating is $0.25 \mathrm{~cm}$. In all the calculations, small imaginary parts (about $0.5 \%$ of the corresponding real parts) were introduced into the elastic constants of the rubber coating to mimic absorption. The resulting complex effective mass densities are shown in Fig. 2(a), where the solid and dashed lines represent the real and imaginary parts of the complex EMD, respectively. It can be seen that the real and the imaginary parts of the EMD display features typical of the Kramers-
Kronig relation. For the real part of the EMD, we observe two frequency regions where it becomes negative, corresponding almost exactly to the two stop bands of the sonic crystal structures reported in Ref. 1 . The static limit of the EMD, i.e., $\rho_{e}^{0}$, is recovered at very low frequency far away from the resonance frequencies of the coated spheres. We have also calculated the vibrating amplitude of the spheres as a function of the frequencies, which is also complex as shown in Fig. 2(b), with the solid line corresponding to the real part and the dashed line corresponding to the imaginary part. It is observed that around $f=378 \mathrm{~Hz}$, there exists a resonance in which the displacements of the spheres are very large and experience a very sharp change from the in-phase state to the out-of-phase state. The first negative EMD frequency region in Fig. 2(a) corresponds to the out-of-phase region labeled as $B$ in Fig. 2(b). It is also interesting to note that in the inphase resonance region labeled A in Fig. 2(b), there exists a large EMD enhancement [see Fig. 2(a)]. From Fig. 2(b), we also observe a big change of $U$ at $f=1333 \mathrm{~Hz}$ [inbetween $\mathrm{C}$ and D in Fig. 2(b)], which corresponds to the second negative EMD region, but the magnitude is small, and thus the negative EMD effect at the second region cannot be attributed to the vibration of the spheres. To further clarify the physics at the first negative EMD region and the origin of the second negative EMD region, we calculate the displacement field distribution along a general radial direction (say, the line from $a$ to $b$ at an angle $\theta=\pi / 4$ shown in Fig. 1) in the coating layer, for four frequencies: A at $f=375 \mathrm{~Hz}, \mathrm{~B}$ at $f=380 \mathrm{~Hz}, \mathrm{C}$ at $f=1330 \mathrm{~Hz}$, and D at $f=1335 \mathrm{~Hz}$. The results are shown in Figs. 3(a)-3(d), with the solid lines denoting the real parts and the dashed line denoting the imaginary parts. We observed that at frequencies labeled A and B, the spheres are vibrating with big amplitude, and the coating around them just follow their motion; while at frequencies $\mathrm{C}$ and $\mathrm{D}$, there are obviously resonant modes inside the coating with displacement amplitude taking maximum values locally in the coating medium, while the vibration of the spheres is very small. We see that all the physics brought out by the simple model is consistent with that by the exact multiplescattering calculation as was done in Ref. 1.

\section{TWO-DIMENSIONAL SYSTEMS}

We now consider a 2D three-component composite material model that is formed with hard cylinders concentrically coated with a soft material. These coated cylinders are embedded in a matrix material. A cross section of the basic building block can be also represented by Fig. 1, where medium 3 now labels the hard cylinder. Suppose that a long wavelength elastic wave is traveling along the $x-y$ plane perpendicular to the axis of the cylinder. The cylinder, as well as the embedding medium (i.e., medium 1) locally, is vibrating around the cylinder axis. Suppose that the vibration is along the $x$ direction (the arrow direction in Fig. 1), the motion of the cylinder satisfies

$$
-m_{3} \omega^{2} U=\int_{0}^{2 \pi}\left(\tau_{r r} \cos \theta-\tau_{r \theta} \sin \theta\right) a l d \theta,
$$

where $U$ is the displacement of the cylinder, $m_{3}$ is the mass of the cylinder expressed as $\rho_{3} \pi a^{2} l$ with $\rho_{3}$ being the density, 

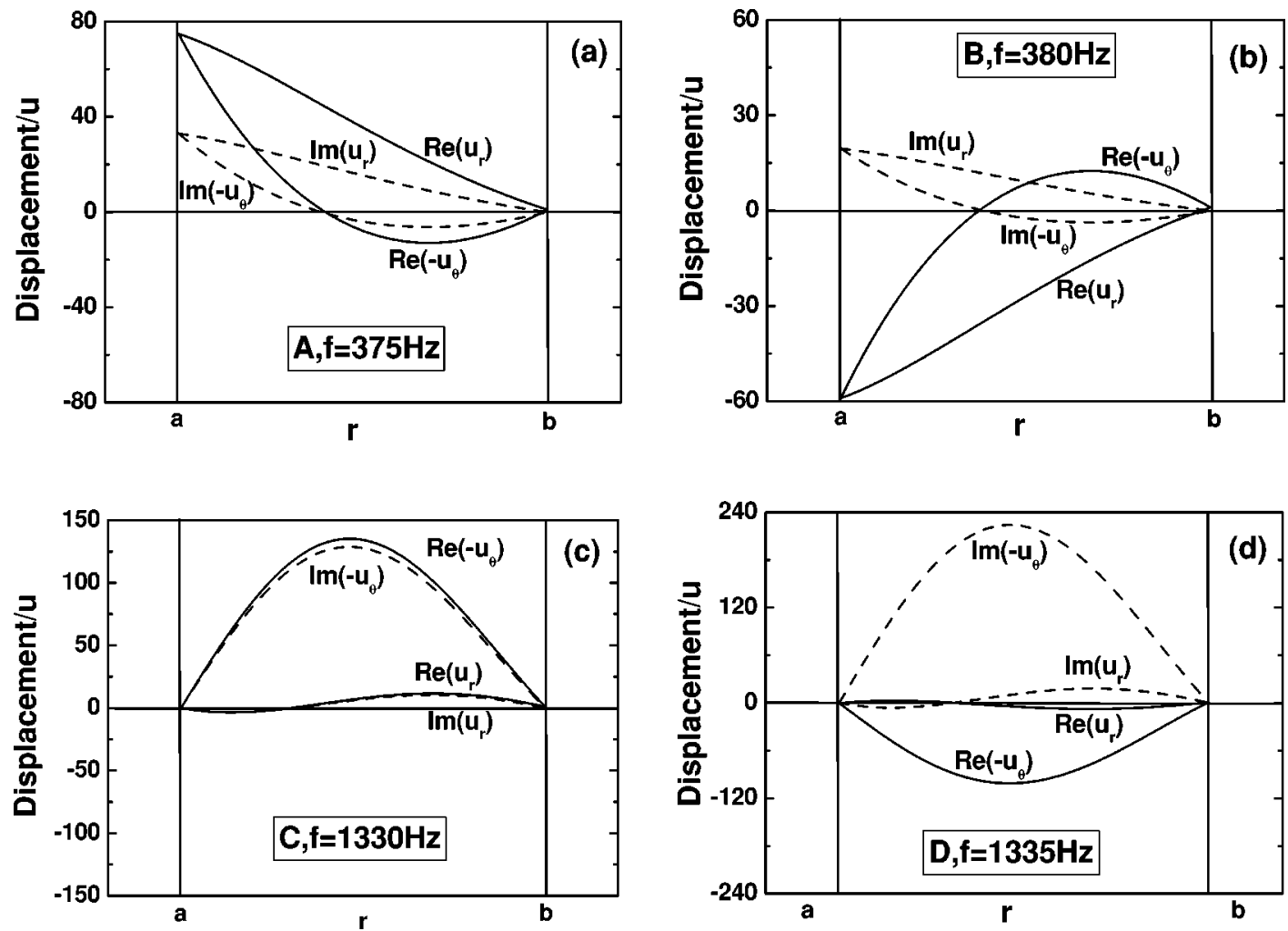

FIG. 3. The displacement fields (normalized to the displacement amplitude $u$ in medium 1) along the line from $a$ to $b$ in the coating layer as shown in Fig. 1 at four frequencies: (a) at A, $f=375 \mathrm{~Hz}$; (b) at $\mathrm{B}, f=380 \mathrm{~Hz}$, (c) at $\mathrm{C}, f=1330 \mathrm{~Hz}$, and (d) at $\mathrm{D}, f=1335 \mathrm{~Hz}$. The solid line and dashed line represent the real part and imaginary part, respectively. From panels (a), and (b), we see that the displacement is largest at $a$, which is the boundary with the core, showing that the core is vibrating strongly at the low frequency resonance. From panels (c) and (d), which correspond to the higher frequency resonance, the displacement peaks inside the coating layer. The change of phase from below to above the resonance is evident in these panels.

$a$ is the radius, and $l$ is the length of the cylinders. Here, $\tau_{r r}$ and $\tau_{r \theta}$ are stress components in medium 2 in a cylindrical coordinate system, and the integration is performed on the surface of the cylinder. Again in medium 2, the motion of the medium should be described by the elastic wave equation [i.e., Eq. (2)]. The mirror symmetry about the $x$ axis indicates that the displacement in medium 2 may be expressed in terms of potential functions as

$$
\mathbf{u}=\boldsymbol{\nabla} \Phi+\boldsymbol{\nabla} \times(\hat{\mathbf{z}} \Psi),
$$

or, in the present case, as

$$
\begin{gathered}
\Phi=\sum_{n=0}^{\infty}\left[A_{n 1} J_{n}(\alpha r)+B_{n 1} N_{n}(\alpha r)\right] \cos (n \theta), \\
\Psi=\sum_{n=0}^{\infty}\left[A_{n 2} J_{n}(\beta r)+B_{n 2} N_{n}(\beta r)\right] \sin (n \theta),
\end{gathered}
$$

where $J_{n}(x)$ and $N_{n}(x)$ are the $n$th order Bessel function and Neumann function. Similar to the derivation in the 3D case (for the details, please see the Appendix), we obtain the expressions for the EMD for this 2D composite as

$$
\rho_{e}=\phi_{1} \rho_{1}+\left(\phi_{2}+\phi_{3}\right) \rho_{2}\left[g_{1}(\omega)-g_{2}(\omega) \frac{g(\omega)}{R(\omega)-\frac{\rho_{3}}{\rho_{2}}}\right],
$$

where $\rho_{1}$ and $\rho_{2}$ are the mass densities for the matrix and the coating materials, and $\phi_{1}, \phi_{2}$, and $\phi_{3}$ are the volume filling fractions of the matrix, the coating, and the core materials, respectively, satisfying $\phi_{1}+\phi_{2}+\phi_{3}=1$. It can also be shown that at the low-frequency limit, $\rho_{e}$ reduces to $\rho_{e}^{0}=\phi_{1} \rho_{1}+\phi_{2} \rho_{2}+\phi_{3} \rho_{3}$, i.e., the average density. The expressions for the frequency dependent $R(\omega), g(\omega), g_{1}(\omega)$, and $g_{2}(\omega)$ are given in the Appendix.

To demonstrate that the EMD may also become negative at some frequency regime, we calculate the EMD for a threecomponent system composed of the same materials as the preceding 3D system, that is, the rubber-coated lead cylinders embedded in epoxy. The filling fraction for coated cylinders is $40 \%$, the cylinder radius is $0.5 \mathrm{~cm}$, and the thickness of the rubber layer is $0.25 \mathrm{~cm}$. The calculated EMD is shown in Fig. 4(a), where we also observe two frequency regions where the real part of the EMD becomes negative. The corresponding vibration amplitude of the cylinders core is shown in Fig. 4(b), where we see that the first negative EMD region is again from the resonance of the lead 

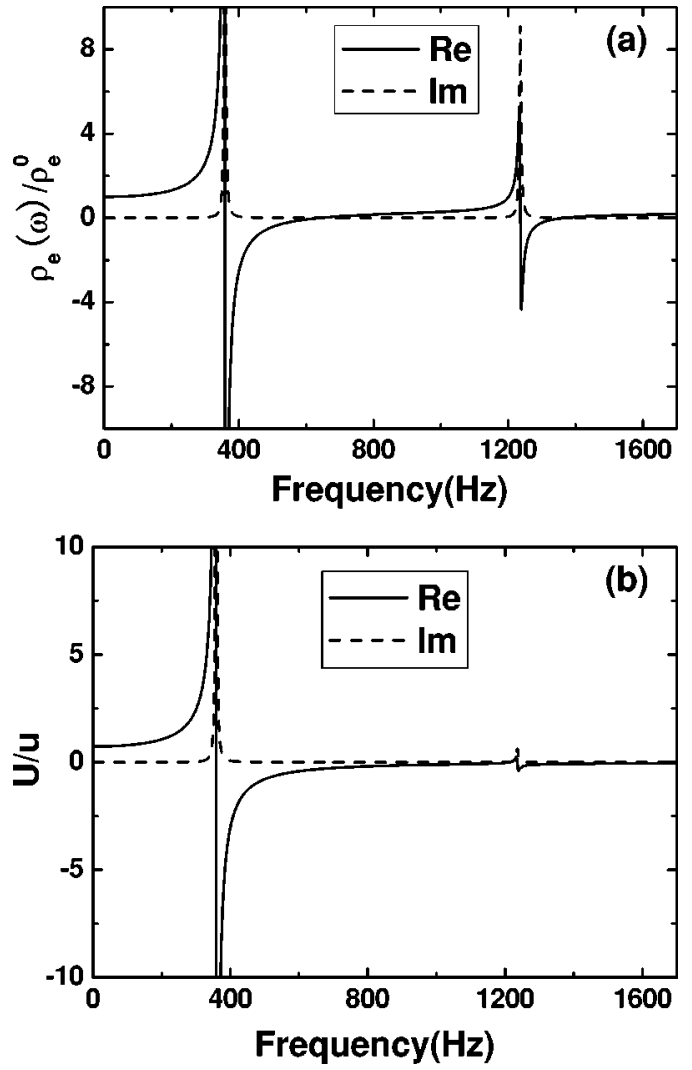

FIG. 4. (a) The $\operatorname{EMD(s)~and~(b)~the~displacement~of~the~core~}$ cylinder for rubber-coated lead cylinders embedded in an epoxy matrix. The solid line and dashed line represent the real part and imaginary part, respectively. The filling fraction for coated cylinders is $40 \%$, the radius of the lead cylinder is $5.0 \mathrm{~mm}$, and the coating thickness is $2.5 \mathrm{~mm}$.

cylinder. The second negative EMD region is from the rubber layer resonance. The physics is thus qualitatively the same as that in the $3 \mathrm{D}$ case.

\section{CONCLUDING REMARKS}

In this paper we show that a simple analytic model can account for low-frequency wave propagation phenomena in a certain class of three-component composite materials ${ }^{1}$ that exhibits sonic band gaps due to resonance. The origin of the gap and the dispersion near the gap can be described by adopting frequency dependent effective mass density that becomes negative near the resonance frequencies of the embedded coated objects. The derivation here captures the essence of the physics, and highlights the importance of the cladding layer. Indeed, the resonance gaps will disappear if the coating is removed or replaced by a stiff material. ${ }^{1}$

\section{ACKNOWLEDGMENTS}

This work was supported in part by Hong Kong RGC Grant Nos. HKUST6138/00P and HKUST6143/00P and China NSFC Grant Nos. 10174054 and 50425206. C.T.C. thanks Dr. Waldemar Maysenhoelder for stimulating discussions and his preprint. ${ }^{4}$

\section{APPENDIX}

In the appendix, we give the derivation of the EMD for the $2 \mathrm{D}$ three-component composite in detail. The displacement continuity at the boundaries gives

$$
\begin{gathered}
\left.u_{r}\right|_{r=b}=u \cos \theta, \\
\left.u_{\theta}\right|_{r=b}=-u \sin \theta, \\
\left.u_{r}\right|_{r=a}=U \cos \theta, \\
\left.u_{\theta}\right|_{r=a}=-U \sin \theta,
\end{gathered}
$$

where $u$ is the local displacement of medium 1. From Eqs. (39) and (40), we see that the expansions naturally truncate for $n>1$, and we immediately get

$$
\begin{aligned}
& E_{11} A_{11}+E_{12} A_{12}+E_{13} B_{11}+E_{14} B_{12}=a U, \\
& E_{21} A_{11}+E_{22} A_{12}+E_{23} B_{11}+E_{24} B_{12}=a U, \\
& E_{31} A_{11}+E_{32} A_{12}+E_{33} B_{11}+E_{34} B_{12}=b u, \\
& E_{41} A_{11}+E_{42} A_{12}+E_{43} B_{11}+E_{44} B_{12}=b u,
\end{aligned}
$$

where $E_{i j} \mathrm{~s}$ are defined as

$$
\begin{gathered}
E_{11}=\alpha a J_{0}(\alpha a)-J_{1}(\alpha a), \\
E_{12}=J_{1}(\beta a), \\
E_{13}=\alpha a N_{0}(\alpha a)-N_{1}(\alpha a), \\
E_{14}=N_{1}(\beta a), \\
E_{21}=J_{1}(\alpha a), \\
E_{22}=\beta a J_{0}(\beta a)-J_{1}(\beta a), \\
E_{23}=N_{1}(\alpha a), \\
E_{24}=\beta a N_{0}(\beta a)-N_{1}(\beta a), \\
E_{31}=\alpha b J_{0}(\alpha b)-J_{1}(\alpha b), \\
E_{32}=J_{1}(\beta b), \\
E_{33}=\alpha b N_{0}(\alpha b)-N_{1}(\alpha b), \\
E_{34}=N_{1}(\beta b), \\
E_{41}=J_{1}(\alpha b), \\
E_{42}=\beta b J_{0}(\beta b)-J_{1}(\beta b), \\
E_{44}=E_{43}=N_{1}(\alpha b), \\
N_{0}(\beta b)-N_{1}(\beta b) .
\end{gathered}
$$

Denoting the inverse of matrix $E$ by $T$, we get 


$$
\begin{aligned}
& A_{11}=\left(T_{11}+T_{12}\right) a U+\left(T_{13}+T_{14}\right) b u, \\
& A_{12}=\left(T_{21}+T_{22}\right) a U+\left(T_{23}+T_{24}\right) b u, \\
& B_{11}=\left(T_{31}+T_{32}\right) a U+\left(T_{33}+T_{34}\right) b u, \\
& B_{12}=\left(T_{41}+T_{42}\right) a U+\left(T_{43}+T_{44}\right) b u .
\end{aligned}
$$

We finally obtain

$$
U=-\frac{\frac{b}{a} g(\omega)}{R(\omega)-\frac{\rho_{3}}{\rho_{2}}} u,
$$

where $R(\omega)$ and $g(\omega)$ are expressed as

$$
\begin{aligned}
R(\omega)= & \left(T_{11}+T_{12}\right) J_{1}(\alpha a)+\left(T_{21}+T_{22}\right) J_{1}(\beta a) \\
& +\left(T_{31}+T_{32}\right) N_{1}(\alpha a)+\left(T_{41}+T_{42}\right) N_{1}(\beta a), \\
g(\omega)= & \left(T_{13}+T_{14}\right) J_{1}(\alpha a)+\left(T_{23}+T_{24}\right) J_{1}(\beta a) \\
& +\left(T_{33}+T_{34}\right) N_{1}(\alpha a)+\left(T_{43}+T_{44}\right) N_{1}(\beta a),
\end{aligned}
$$

respectively. Resonance occurs at $R(\omega)=\rho_{3} / \rho_{2}$.

The final expressions for $A_{11}, A_{12}, B_{11}, B_{12}$ are

$$
\begin{aligned}
& A_{11}=\left[\left(T_{13}+T_{14}\right)-\left(T_{11}+T_{12}\right) \frac{g(\omega)}{R(\omega)-\frac{\rho_{3}}{\rho_{2}}}\right] b u, \\
& A_{12}=\left[\left(T_{23}+T_{24}\right)-\left(T_{21}+T_{22}\right) \frac{g(\omega)}{R(\omega)-\frac{\rho_{3}}{\rho_{2}}}\right] b u, \\
& B_{11}=\left[\left(T_{33}+T_{34}\right)-\left(T_{31}+T_{32}\right) \frac{g(\omega)}{R(\omega)-\frac{\rho_{3}}{\rho_{2}}}\right] b u, \\
& B_{12}=\left[\left(T_{43}+T_{44}\right)-\left(T_{41}+T_{42}\right) \frac{g(\omega)}{R(\omega)-\frac{\rho_{3}}{\rho_{2}}}\right] b u .
\end{aligned}
$$

The force acting on the coated cylinder by medium 1 may be expressed as

$$
F_{23}=\int_{0}^{2 \pi}\left(\tau_{r r} \cos \theta-\tau_{r \theta} \sin \theta\right) l b d \theta,
$$

which is obtained as

$$
\begin{gathered}
F_{23}=-\pi b l \rho_{2} \omega^{2}\left[A_{11} J_{1}(\alpha b)+A_{12} J_{1}(\beta b)\right. \\
\left.+B_{11} N_{1}(\alpha b)+B_{12} N_{1}(\beta b)\right] \\
=-\pi b^{2} l \rho_{2} \omega^{2}\left[g_{1}(\omega)-g_{2}(\omega) \frac{g(\omega)}{R(\omega)-\frac{\rho_{3}}{\rho_{2}}}\right] u,
\end{gathered}
$$

and

$$
\begin{aligned}
g_{1}(\omega)= & \left(T_{13}+T_{14}\right) J_{1}(\alpha b)+\left(T_{23}+T_{24}\right) J_{1}(\beta b) \\
& +\left(T_{33}+T_{34}\right) N_{1}(\alpha b)+\left(T_{43}+T_{44}\right) N_{1}(\beta b),
\end{aligned}
$$

$$
\begin{aligned}
g_{2}(\omega)= & \left(T_{11}+T_{12}\right) J_{1}(\alpha b)+\left(T_{21}+T_{22}\right) J_{1}(\beta b) \\
& +\left(T_{31}+T_{32}\right) N_{1}(\alpha b)+\left(T_{41}+T_{42}\right) N_{1}(\beta b) .
\end{aligned}
$$

We note that the force between the coated cyliner and the embedding medium, as expressed by $F_{23}$, is a consequence of the resonance behavior. Far away from the resonance and in the low-frequency limit, $F_{23}$ reduces to $F_{23}^{0}=-\left(m_{2}\right.$ $\left.+m_{3}\right) \omega^{2} u$, with $m_{2}$ being the mass of the coating. $F_{23}^{0}$ is the force acting on the coated cylinder if we ignore the resonance. We can define the effective mass density for the coated cylinder through $F_{23}$ by

$$
-\rho_{23}^{e} V_{23} \omega^{2} u=F_{23},
$$

where $V_{23}=\pi b^{2} l$ is the volume of the coated cylinder. We obtain

$$
\rho_{23}^{e}=\rho_{2}\left[g_{1}(\omega)-g_{2}(\omega) \frac{g(\omega)}{R(\omega)-\frac{\rho_{3}}{\rho_{2}}}\right]
$$

The effective mass density for the whole local unit can be defined as

$$
\begin{aligned}
\rho_{e} & =\phi_{1} \rho_{1}+\left(\phi_{2}+\phi_{3}\right) \rho_{23}^{e} \\
& =\phi_{1} \rho_{1}+\left(\phi_{2}+\phi_{3}\right) \rho_{2}\left[g_{1}(\omega)-g_{2}(\omega) \frac{g(\omega)}{R(\omega)-\frac{\rho_{3}}{\rho_{2}}}\right],
\end{aligned}
$$

where $\phi_{1}, \phi_{2}$, and $\phi_{3}$ are the filling fractions for media 1,2, and 3 , respectively, satisfying $\phi_{1}+\phi_{2}+\phi_{3}=1$. It can be seen that at the low-frequency limit, $\rho_{e}$ reduces to $\rho_{e}^{0}=\phi_{1} \rho_{1}$ $+\phi_{2} \rho_{2}+\phi_{3} \rho_{3}$, i.e., the average density. 
${ }^{1}$ Z. Liu, X. Zhang, Y. Zhu, Y. Mao, C. T. Chan, and P. Sheng, Science 289, 1734 (2000); Z. Liu, C. T. Chan, and P. Sheng, Phys. Rev. B 65, 165116 (2002).

${ }^{2}$ See, e.g., C. Kittel, Introduction of Solid State Physics, 6th ed. (Wiley, New York, 1991).

${ }^{3}$ C. Goffaux, J. Sanchez-Dehesa, A. L. Yeyati, Ph. Lambin, A. Khelif, J. O. Vasseur, and B. Djafari-Rouhani, Phys. Rev. Lett. 88, 225502 (2002); C. Goffaux and J. Sanchez-Dehesa, Phys. Rev. B 67, 144301 (2003).
${ }^{4}$ W. Maysenhoelder (unpublished).

${ }^{5}$ See, for example, Z. Liu, C. T. Chan, P. Sheng, A. L. Goertzen, and J. H. Page, Phys. Rev. B 62, 2446 (2000); I. E. Psarobas, N. Stefanou, and A. Modinos, ibid. 62, 278 (2000).

${ }^{6}$ See for example, P. M. Morse and H. Feshbach, Methods of Theoretical Physics (McGraw-Hill, New York, 1953).

${ }^{7}$ K. F. Graff, Wave Motion in Elastic Solids (Dover, New York, 1991). 